m35.002

\section{Kirkpatrick-Baez mirrors used in high resolution $x$-ray microscopy, tomography and fluorescence analysis}

$\underline{\text { Peter Cloetens }}^{\mathrm{a}}$, Sylvain Bohic ${ }^{\mathrm{a}, \mathrm{b}}$, Christine Borel ${ }^{\mathrm{a}}$, Olivier Hignette $^{\mathrm{a}}$, Greg Johnson ${ }^{\mathrm{a}, \mathrm{c}}$, Wolfgang Ludwig ${ }^{\mathrm{a}, \mathrm{d}}$, Rajmund Mokso $^{\text {a }}$, Christian Morawe ${ }^{a}$, Richard Ortega ${ }^{e}$, Remi Tucoulou ${ }^{\mathrm{a}}$,

${ }^{a}$ ESRF, Grenoble. ${ }^{b}$ INSERM U-647, UJF. ${ }^{c}$ University of Manchester. ${ }^{d}$ GEMPPM, INSA de Lyon. ${ }^{e}$ CNRS, Université Bordeaux. E-mail: cloetens@esrf.fr

\section{Keywords: X-ray focusing, tomography, X-ray microfluorescence}

The combination of a very brilliant X-ray source and state-ofthe-art X-ray optics opens the way to nano-focusing applications. Dynamically bent graded multilayers set in the KB-geometry are developed at the ESRF as an extremely efficient device to focus undulator radiation to spots below $100 \mathrm{~nm}$ [1]. The smallest 2D focus reached so far with this device is of the order of $80 \mathrm{~nm}$ in both directions. This has been achieved both on a long, coherent beamline and on a shorter beamline using the concept of a secondary source. The first mirror of the system, coated with a graded multilayer, plays both the role of vertical focusing device and monochromator, resulting in a very high flux (a few $10^{11}$ photons/s) and medium monochromaticity $\left(\Delta \mathrm{E} / \mathrm{E} \sim 10 \mathrm{p}^{2}\right)$. Two different fields benefit particularly from the combination small focus / high flux: projection microscopy (magnified tomography) and X-ray fluorescence mapping. In projection microscopy the sample is set at a small distance $(10-100 \mathrm{~mm})$ downstream or upstream of the focus and a magnified Fresnel diffraction pattern is recorded on a medium resolution detector set at a large distance (several meters) from the focus. This approach allows to overcome the spatial resolution limit imposed by the detector in the parallel beam geometry. It was used to give direct evidence of nanometric invasion-like grain boundary penetration in the $\mathrm{Al} / \mathrm{Ga}$ system [2]. For tomography, scans are acquired at different focussample distances. This yields variable magnifications, but also different effective propagation distances ( equal to the focussample distance). The principal difficulties in this approach are related to the mirror imperfections and the pronounced refraction effects at the sample edges when going to sub-100 $\mathrm{nm}$ pixel sizes. Satisfactory results were however obtained in local tomography mode on aluminium alloys. A correction for mirror figure error and a phase retrieval method adapted to the imaging setup are incorporated in the tomograhic procedure [3]. In fluorescence imaging the sample is scanned through the focal plane while the spectrum of the emitted fluorescence is recorded with an energy dispersive detector. This rich probe, complementary to transmission imaging, provides element specific information and allows to image and quantify trace elements. The possibility to apply this method to the field of neurochemistry at the sub-cellular level has been demonstrated. Finally, the best one dimensional focus reached so far with reflective multilayer optics is $40 \mathrm{~nm}$. The ultimate limits of this focusing approach will be discussed.

[1] Hignette O.; Cloetens P.; Rostaing G.; Bernard P.; Morawe C. Rev. Sci. Instrum. 2005, 76, 063709.

[2] Pereiro E.; Ludwig W.; Bellet D.; Cloetens P.; Lemaignan C. Phys. Rev. Lett. 2005, 95, 215501.

[3] Mokso R.; Cloetens P.; Maire E.; Ludwig W.; Buffière J.-Y. submitted to Appl. Phys. Lett. m35.003

\section{Hard X-Ray Nanoprobe with Refractive X-Ray Lenses}

Christian Schroer, ${ }^{\text {a }}$ Olga Kurapova, ${ }^{\mathrm{b}}$ Jens Patommel, ${ }^{\mathrm{b}}$ Pit Boye, ${ }^{\mathrm{b}}$ Jan Feldkamp, ${ }^{\mathrm{b}}$ Bruno Lengeler, ${ }^{\mathrm{b}}$ Manfred Burghammer, ${ }^{\mathrm{c}}$ Christian Riekel, ${ }^{\mathrm{c}}$ Laszlo Vincze ${ }^{\mathrm{d}}$

${ }^{a}$ HASYLAB at DESY, Notkestr. 85, D-22607 Hamburg. ${ }^{b}$ II. Physikalisches Institut, Aachen University, D-52056 Aachen, Germany. ${ }^{c}$ European Synchrotron Radiation Facility ESRF, BP 220, F-38043 Grenoble, France. ${ }^{d}$ Department of Analytical Chemistry, Ghent University, Krijgslaan 281 S12, B-9000 Ghent, Belgium.E-mail: christian.schroer@desy.de

\section{Keywords: X-ray nanobeam, nanodiffraction, refractive $X$-ray lenses}

A hard x-ray nanoprobe based on refractive x-ray lenses allows one to apply with high spatial resolution hard x-ray analytical techniques, such as diffraction or fluorescence analysis. This is particularly useful to obtain local structural and chemical information from heterogeneous samples and can be applied e. g. in physics, chemistry, nano-, materials, environmental, and life science. Using nanofocusing lenses (NFLs) [1-2], a nanobeam with a lateral extension down to $50 \times 50 \mathrm{~nm}^{2}$ is currently feasible at third generation synchrotron radiation sources in the hard $\mathrm{x}$-ray range. The beam divergence of about $1 \mathrm{mrad}$ is sufficient for many diffraction experiments. The beam size can be expected to be reduced to below $10 \mathrm{~nm}$ in the future [3]. The coherence properties of the nanobeam are discussed in view of diffraction from small objects with coherent radiation.

[1] C. G. Schroer, et al., Appl. Phys. Lett. 82, 1485 (2003).

[2] C. G. Schroer, et al., Appl. Phys. Lett. 87, 124103 (2005)

[3] C. G. Schroer, B. Lengeler, Phys. Rev. Lett. 94, 054802 (2005). 